Danniela Britto de Carvalho ${ }^{a}$

Tânia Maria de Araújo ${ }^{\mathrm{a}}$

Kionna Oliveira Bernardes ${ }^{b}$

\section{Transtornos mentais comuns em trabalhadores da Atenção Básica à Saúde}

\author{
Common mental disorders in primary health care workers
}

a Universidade Estadual de Feira de Santana, Programa de Pós-Graduação em Saúde Coletiva., Feira de Santana, BA, Brasil.

b Universidade Federal da Bahia, Instituto de Ciências da Saúde. Salvador, Bahia, Brasil.

\section{Contato:}

Danniela Britto de Carvalho

E-mail:

danniela18.carvalho@yahoo.com.br

Este trabalho foi financiado pela Fundação de Amparo à Pesquisa do Estado de São Paulo (Fapesp), pelo Ministério da Saúde (Edital 04/2009 - PPSUS) e pelo CNPq (Edital Universal 2010).

Este trabalho baseia-se na dissertação de mestrado de Danniela Brito de Carvalho, intitulada "Transtornos mentais comuns em trabalhadores da atenção básica à saúde”, defendida em 2012 no Programa de Pós-Graduação em Saúde Coletiva da Universidade Estadual de Feira de Santana.

Trabalho não apresentado em reunião científica.

Recebido: 14/05/2015

Revisado: 28/03/2016

Aprovado: 30/03/2016

\section{Resumo}

Introdução: os trabalhadores da saúde podem ser acometidos por doenças relacionadas ao trabalho. A literatura registra que os transtornos mentais constituem importante fator de adoecimento entre esses trabalhadores. Objetivo: avaliar a prevalência de transtornos mentais comuns (TMC) e os fatores sociodemográficos, de estilo de vida e de trabalho, associados à sua ocorrência entre trabalhadores da Atenção Básica à Saúde. Métodos: estudo exploratório, de corte transversal, com amostra estratificada proporcional de trabalhadores das Unidades Básicas de Saúde, Unidades de Saúde da Família, equipe de Vigilância Epidemiológica e Núcleo de Apoio à Saúde da Família (Nasf). Resultados: a prevalência global de TMC foi de 22,9\%; entre os trabalhadores do Nasf atingiu $31,6 \%$. Observou-se associação positiva e estatisticamente significante $(p \leq 0,05)$ entre TMC e sexo feminino, qualidade de vida regular/ruim/muito ruim, estado de saúde regular/ruim/muito ruim, pressão do tempo, insatisfação com o trabalho e elevadas demandas psicológicas. Discussão: devem ser priorizadas ações sobre os aspectos relacionados ao gênero, ao estado de saúde, qualidade de vida e, especialmente, sobre os aspectos da organização do trabalho, como a pressão do tempo, satisfação no trabalho e demanda psicológica envolvida.

Palavras-chave: transtornos mentais; Atenção Primária à Saúde; pessoal de saúde.

\begin{abstract}
Introduction: health care workers may be exposed to work-related diseases. The literature reports that mental disorders are important causes of illness among these workers. Objective: to evaluate the prevalence of Common Mental Disorders (CMD) and sociodemographic, lifestyle and working life factors associated with CMD occurrence among Primary Health Care Services workers. Methods: exploratory, cross-sectional study with a proportional stratified sample of workers from Primary Health Units, Family Health Units, Epidemiological Surveillance Team and Support Center for Family Health (NASF). Mental health was assessed by the Self-Reporting Questionnaire (SRQ-20) and the psychosocial aspects of work by the Job Content Questionnaire (JCQ). Results: the global CMD prevalence was $22.9 \%$; among workers from NASF it reached $31.6 \%$. Positive association, statistically significant ( $p \leq 0.05$ ), was observed between CMD and women, regular/bad/very bad quality of life, regular/bad/very bad health status, time pressure, dissatisfaction with work and high psychological demands. Discussion: actions related to gender, health status, quality of life and especially to work organization aspects, such as time pressure, psychological demands and job satisfaction should be prioritized.
\end{abstract}

Keywords: mental disorders; primary health care; health care personnel. 


\section{Introdução}

Os transtornos mentais e comportamentais são condições clínicas caracterizadas por alterações nos pensamentos e nas emoções ou por comportamentos relacionados à angústia pessoal e/ou à deterioração do funcionamento psíquico, tendo efeitos deletérios, atingindo não somente o indivíduo, mas a família e a comunidade ${ }^{1}$. São prevalentes em todo o mundo: estima-se que $10 \%$ dos adultos apresenta tais condições e que $25 \%$ da população mundial manifesta, pelo menos, um transtorno mental ao longo da vida.

Esses transtornos podem ser causados por diversos fatores, destacando-se aqueles relacionados ao ambiente profissional, como baixo nível de controle sobre o próprio trabalho, elevadas demandas psicológicas e baixo apoio social ${ }^{2}$. Os transtornos mentais relacionados ao trabalho têm sido considerados problema de saúde pública em função de sua alta prevalência em profissionais e de suas consequências como o absenteísmo, incapacidades para o trabalho e aposentadoria precoce ${ }^{3}$. Apesar de serem prevalentes entre os trabalhadores, esses transtornos não são reconhecidos com frequência nos atendimentos clínicos $^{4-6}$, sendo subdimensionados. Setores em que há demandas psicossociais muito elevadas devido às características estressantes do tipo de trabalho realizado - como na área da saúde -, os trabalhadores estão mais vulneráveis ao adoecimento psíquico e sofrimento.

No Brasil, a investigação da relação entre saúde e trabalho em saúde foi mais tardia do que em outras categorias profissionais. Os estudos existentes, embora esparsos, incompletos e fragmentados, indicam aumento na frequência das doenças, dos acidentes ocupacionais e de transtornos mentais comuns (TMC) entre os trabalhadores da saúde. Esses estudos indicam também contribuição importante no adoecimento psíquico de características do ambiente de trabalho, do contexto e das condições gerais nas quais os serviços são oferecidos e da gestão do trabalho nos estabelecimentos de saúde ${ }^{4,5,7}$. Esses achados reforçam a necessidade de diagnóstico mais efetivo da situação existente e de verificação de seus fatores determinantes.

Considerando que a maioria dos estudos no Brasil que avaliaram saúde mental e trabalho no setor saúde investigou trabalhadores no contexto hospitalar, torna-se relevante a análise desses aspectos no âmbito dos serviços de atenção básica à saúde, de modo a fornecer informações sobre a magnitude dos transtornos mentais nesse grupo e os fatores associados à sua ocorrência.

A Atenção Básica constitui a principal porta de entrada dos usuários ao sistema de saúde, e se orienta pelos princípios da universalidade, acessibilidade, integralidade, responsabilização, humanização, equidade, participação social, coordenação do cuidado, do vínculo e continuidade da atençãa ${ }^{8}$. Para atender a esses princípios, a Atenção Básica estabelece exigências diversificadas aos trabalhadores $^{9}$; exigências muito distintas daquelas existentes na atenção hospitalar. $\mathrm{O}$ trabalho é desenvolvido através de práticas gerenciais, sanitárias, composta por um conjunto de ações individuais e coletivas, abrangendo a promoção e a proteção da saúde, a prevenção de agravos, o diagnóstico, o tratamento, a reabilitação e a manutenção da saúde, executadas sob a forma do trabalho em equipe, dirigidas a populações de áreas territoriais específicas, das quais possui responsabilidade sanitária, levando em consideração a dinamicidade do território, assumindo-se a responsabilidade de resolver os problemas mais frequentes e relevantes do território ${ }^{8}$.

A estruturação do novo modelo de atenção à saúde, focalizado na estratégia de saúde da família, estabeleceu um conjunto de novos contextos laborais, bem como a emergência de novos grupos ocupacionais. Os espaços laborais foram ampliados e se estenderam para além das unidades de saúde, chegando até o domicílio das populações atendidas. Do ponto de vista das equipes de trabalho, também foram introduzidas mudanças substantivas com ênfase nas intervenções multiprofissionais.

Nesse sentido, os Núcleos de Apoio à Saúde da Família (Nasf) foram criados para oferecer apoio multidisciplinar ao mapeamento de agravos e prevenção realizados pelo Programa Saúde da Família (PSF), sendo estruturado na perspectiva de atuar numa lógica de corresponsabilização e de gestão integrada do cuidado, por meio de atendimentos e projetos terapêuticos voltados aos usuários de modo a considerar sua singularidade ${ }^{10}$. No entanto, as exigências de atuação sob novos moldes não têm sido acompanhadas pelo adequado processo de formação e preparação dos trabalhadores, produzindo situações geradoras de sofrimento, que se referem, por exemplo, no caso do Nasf, à definição difusa do que representa o papel de "apoio" e ao emprego de novas tecnologias de cuidado em saúde voltado para a perspectiva da interdisciplinaridade ${ }^{11}$. Problemas semelhantes podem ser destacados também com relação aos demais trabalhadores da saúde, especialmente aqueles de inserção recente no modelo de atenção, como os agentes comunitários de saúde. Essas alterações, por sua vez, introduziram novas exposições ocupacionais cujos impactos na saúde dos trabalhadores ainda não são bem conhecidos, necessitando, assim, ser melhor exploradas.

A necessidade de pesquisas envolvendo os profissionais da atenção básica evidencia-se especialmente 
em função do tipo de trabalho prestado, nas responsabilidades decorrentes do atendimento integral que deve ser ofertado à população, bem como pelas exigências de ações intersetoriais que articulam o trabalho executado a outros níveis de complexidade no sistema de saúde ${ }^{12}$. Melhorar as condições de vida e de trabalho dos trabalhadores da saúde poderá proporcionar impacto positivo nas ações desempenhadas por toda a equipe da Atenção Básica, com potencialidade para melhorar a assistência à saúde das populações assistidas ${ }^{13}$. A expansão do número de trabalhadores na atenção básica em todo o país é outro fator importante, uma vez que é crescente o número de equipes de saúde da família e de Nasf constituídos e, consequentemente, de trabalhadores neles inseridos ${ }^{14}$.

Registra-se, ainda, como justificativa para a realização de estudos sobre a saúde dos trabalhadores da saúde da atenção básica, os marcos regulatórios do Protocolo $\mathrm{n}^{\mathrm{O}}$ 008/2011, de 1 de dezembro de 2011, que estabeleceu as diretrizes da Política Nacional de Promoção da Saúde do Trabalhador do Sistema Único de Saúde e da Política Nacional do Trabalhador e da Trabalhadora (Portaria ${ }^{\circ} 1.823$, de 23 de agosto de 2012), que reforçam a necessidade e a pertinência de políticas específicas para a proteção e a promoção da saúde desses trabalhadores ou estabelecem princípios gerais para ações com foco nas ações de vigilância em saúde do trabalhador.

Portanto, esse conjunto de fatores fortalece a necessidade e a pertinência de análise da situação de saúde mental dos trabalhadores da atenção básica, bem como dos fatores associados ao sofrimento psíquico identificado. Assim, o objetivo deste estudo foi avaliar a prevalência de transtornos mentais comuns (TMC) e os fatores sociodemográficos, de estilo de vida e de trabalho associados a sua ocorrência entre trabalhadores da Atenção Básica à Saúde.

\section{Materiais e métodos}

\section{Tipo de estudo}

Trata-se de estudo epidemiológico de corte transversal, com finalidade exploratória, para investigar a prevalência de TMC entre os trabalhadores da Atenção Básica à Saúde de Feira de Santana (BA). O estudo foi realizado no período de agosto de 2011 a março de 2012. O estudo piloto ocorreu no mês de junho de 2011, na cidade de Cruz das Almas (BA). Este estudo é um subprojeto da pesquisa "Condições de trabalho, condições de emprego e saúde dos trabalhadores da saúde na Bahia", coordenada pelo Núcleo de Epidemiologia da Universidade Estadual de Feira de Santana (Uefs) em parceria com universidades públicas na Bahia: Universidade Estadual de Santa Cruz (Uesc), Universidade Estadual do Sudoeste da Bahia (Uesb), Universidade Federal do Recôncavo da Bahia (UFRB), Universidade Federal da Bahia (UFBA) e Universidade Federal do Vale do São Francisco (Univasf).

Feira de Santana é a segunda cidade mais populosa do estado da Bahia, sendo também sede da maior região metropolitana do interior do nordeste brasileiro. Ocupa a décima posição em infraestrutura urbana entre as cidades do país; seu Índice de Desenvolvimento Humano (IDH), medido pelas Nações Unidas, é de 0,712 (alto), possuindo uma qualidade de vida superior a $72,24 \%$ dos municípios brasileiros ${ }^{15}$. Com relação ao setor da saúde, possui 318 estabelecimentos de saúde, sendo 207 privados e 111 públicos, dos quais 105 são municipais e 6 estaduais. Feira de Santana encontra-se no nível da gestão plena do sistema de saúde ${ }^{16}$.

\section{População de estudo}

Foram estudados os trabalhadores da Atenção Básica, que estavam distribuídos entre oito áreas de Nasf. A equipe do Nasf, de implantação ainda mais recente, é composta por profissionais de nível superior, com objetivo de estimular práticas saudáveis na comunidade e apoiar as ações com relação a temáticas especializadas.

A atenção básica no município estudado era composta por 16 unidades básicas de saúde (UBS) e 83 unidades de saúde da família (USF). No total, havia 2.446 profissionais trabalhando nessas unidades de saúde, sendo 1.241 nas USF, 856 nas UBS e 349 em ambas (281 agentes de combate às endemias, locados na vigilância epidemiológica, e 68 trabalhadores dos Nasf).

Como o estudo não foi desenvolvido especificamente para análise dos TMC, novo cálculo do tamanho amostral foi realizado a fim de avaliar se o estudo tinha poder suficiente para a investigação de TMC. Para o cálculo da amostra considerou-se o número total de trabalhadores da Atenção Básica (2.446), a prevalência de TMC de $24 \%{ }^{1}$, erro amostral de $3 \%$ e nível de confiança de $95 \%$, obtendo-se tamanho mínimo da amostra a ser estudada de 591 trabalhadores. Como foram estudados 762 trabalhadores, concluiu-se que o estudo tinha poder para a análise proposta.

Para a seleção dos trabalhadores foi feita amostragem estratificada proporcional por área geográfica (áreas de atuação dos Nasf) e categoria profissional. Em cada estrato, os trabalhadores foram sorteados por procedimento aleatório simples com o emprego de lista de números aleatórios. Realizou-se 
levantamento de todos os trabalhadores pertencentes às unidades de saúde e sortearam-se os trabalhadores por categoria profissional. Em seguida, os sorteados foram contatados e convidados a participar da pesquisa. Os contatos foram feitos em seus respectivos locais de trabalho.

Foram incluídos no estudo os trabalhadores que estavam em efetivo exercício profissional, que aceitaram, voluntariamente, responder o instrumento de coleta de dados, e que tinham tempo mínimo de seis meses de trabalho na unidade. Consequentemente, os trabalhadores que não atendiam a esses critérios não foram incluídos no estudo.

Os trabalhadores não encontrados nos locais de trabalho, após três tentativas, foram substituídos por outros trabalhadores, respeitando-se a área geográfica, o grupo ocupacional e o sexo.

\section{Instrumentos de análise}

Aplicou-se instrumento padronizado, composto por oito blocos de questões sobre características sociodemográficas, características do trabalho, incluindo aspectos psicossociais, avaliação do estado de saúde do indivíduo e saúde mental.

A saúde mental foi avaliada pelo Self-Reporting Questionnaire (SRQ-20), que é constituído por vinte questões mensuradas em escala dicotômica que avaliam nível de suspeição de transtornos neuróticos, envolvendo os seguintes grupos de sintomas: humor depressivo/ansioso, sintomas somáticos, decréscimo de energia vital e pensamentos depressivos. O SRQ20 foi testado e validado em contextos ocupacionais no Brasil, apresentando bom desempenho ${ }^{17}$.

Os aspectos psicossociais do trabalho foram mensurados pelo Job Content Questionnaire (JCQ), que avalia níveis de controle do trabalhador sobre o próprio trabalho e as demandas psicológicas envolvidas na atividade laboral ${ }^{18}$.

A coleta de dados foi realizada por equipe de entrevistadores treinados, composta por bolsistas e voluntários de núcleo de pesquisa. Para a entrevista, foram realizados contatos prévios com a coordenação de atenção básica, chefias e com o próprio trabalhador, agendando-se a entrevista. Foram feitas até três tentativas para a realização da entrevista.

Ao receber os questionários, os trabalhadores eram orientados a respondê-los de forma completa, esclarecendo-se dúvidas sobre as questões. Após a devolução, conferiam-se os questionários, a fim de evitar dados perdidos, garantindo maior confiabilidade das informações.

A digitação dos dados foi feita no programa Epidata versão 14.0, em dupla digitação. Para a análise dos dados foram utilizados o programa SPSS for Windows versão 17.0, o Epi Info e o software R.

As variáveis de interesse nesse estudo foram: a) características sociodemográficas: sexo (0 - masculino; 1 - feminino), faixa etária ( $0-\leq 40$ anos; $1-$ 41 anos ou mais), escolaridade (0 - nível superior; 1 - outra escolaridade), situação conjugal (0 - com companheiro; 1 - sem companheiro), ter filhos (0 não; 1 - sim), renda ( $0->2$ salários mínimos; 1 $\leq 2$ salários mínimos); b) hábitos de vida: consumir bebida alcoólica (0 - não; 1 - sim), tabagismo (0 - não; 1 - sim), participação em atividades de lazer (0 - não; 1 - sim), prática de atividade física (0 não; 1 - sim); c) situação geral de saúde: qualidade de vida autorreferida ( 0 - boa/muito boa; 1 - regular/ruim/muito ruim), nível de saúde autorreferido (0 - bom/muito bom; 1 - regular/ruim/muito ruim); c) características do trabalho: tipo de vínculo (0 permanente; 1 - temporário), possuir outro vínculo (0 - sim; 1 - não), jornada semanal ( $0-\leq 40 \mathrm{~h} ; 1$ - acima de $40 \mathrm{~h}$ ), tempo de trabalho (0 - $\leq 5$ anos; $1->5$ anos), pressão do tempo (0 - não; 1 - sim), satisfação com o trabalho (0 - sim; 1 - não), satisfação com a capacidade para o trabalho (0 - sim; 1 - não) e d) aspectos psicossociais do trabalho: demanda psicológica ( 0 - baixa; 1 - alta), controle sobre o próprio trabalho ( 0 - alto; 1 - baixo).

A variável de efeito analisada foram os Transtornos Mentais Comuns (TMC), mensurados pelo SRQ-20, considerando-se como suspeito de TMC os trabalhadores que responderam a sete ou mais perguntas positivamente, com base em estudo de validação conduzido por Santos et al. ${ }^{19}$.

\section{Análise estatística}

Foi feita inicialmente análise descritiva dos trabalhadores estudados com foco nas variáveis socioeconômicas, hábitos de vida e condições de trabalho. Avaliou-se a prevalência de TMC segundo essas variáveis de interesse.

A análise bivariada foi realizada para exploração inicial das associações brutas. Para isso, foram estimadas as razões de prevalência e os intervalos de confiança, adotando-se nível de significância estatística de 5\%.

Também foram estimadas as prevalências de TMC segundo os aspectos psicossociais, por meio do modelo demanda-controle de Karasek - que tem sido amplamente utilizado para investigar associação entre estresse no trabalho e desfechos de saúde. Neste estudo, foram avaliadas as principais escalas (controle e demandas psicológicas), utilizando-se o Job Content Questionnaire (JCQ). Para análise dessas escalas, procedeu-se à composição de grupos 
estabelecidos com base nas medianas do escore de cada uma dessas variáveis, divididos em alto(a) e baixo(a). As prevalências de TMC foram estimadas para demanda psicológica, controle sobre o próprio trabalho e para os tipos de trabalho gerados pela combinação de demanda e controle (trabalho passivo, trabalho ativo, trabalho de alta exigência e de baixa exigência).

A análise de regressão logística múltipla (ARLM) foi conduzida conforme os procedimentos recomendados $^{20}$ e incluiu as seguintes etapas: a) seleção das variáveis a partir dos objetivos do estudo e de revisão de literatura; b) verificação de pressupostos do modelo; c) pré-seleção das variáveis para inclusão na análise através do teste de razão de verossimilhança, adotando um valor de $\mathrm{p} \leq 0,25$; d) ARLM propriamente dita, utilizando procedimento backward. O critério de significância $\mathrm{p}<0,05$ foi adotado para obtenção do modelo final.

A demanda psicológica e o controle sobre o trabalhador foram analisados como variáveis isoladas no modelo completo que foi testado na ARLM. Portanto, os grupos do modelo demanda-controle foram analisados apenas de modo exploratório, na análise bivariada.

A análise de regressão logística produz medidas de razão de odds (OR). Como a prevalência de TMC na população foi maior que $10 \%$, pode haver subestimação da medida de associação (OR). Desse modo, foram estimadas as razões de prevalência a partir dos dados obtidos no modelo final da ARLM. O método Delta foi utilizado para estimar os intervalos de confiança de $95 \%{ }^{21}$.

\section{Aspectos éticos}

O projeto foi submetido ao Comitê de Ética em Pesquisa e aprovado, conforme Resolução 466/2012 do Conselho Nacional de Saúde (Protocolo ${ }^{\circ}$ 081/2009 - CAAE 0086.0059.000-09).

A participação na pesquisa foi voluntária e foi mantido sigilo das informações obtidas. Antes de receberem os questionários, todos os entrevistados leram e assinaram o termo de consentimento livre e esclarecido.

\section{Resultados}

Foram estudados 762 trabalhadores da Atenção Básica de Feira de Santana com predominância dos Agentes Comunitários de Saúde (44,6\%), seguidos dos trabalhadores de enfermagem (19,7\%), agentes de combate a endemias $(13,9 \%)$ e trabalhadores de funções administrativas (recepcionista, vigilante, serviços gerais) $(9,3 \%)$.

Da amostra estudada, 59,8\% atuavam nas USF, 23,8\% nas UBS, 11,9\% na Vigilância Epidemiológica e $4,5 \%$ nos Nasf.

Quanto às características sociodemográficas e aos hábitos de vida, predominou o sexo feminino, população jovem ( $\leq 40$ anos), sem nível superior, com companheiro, com filhos, com renda de até dois salários mínimos, sendo a média de R \$979,00 com mínimo de $\mathrm{R} \$$ 400,00 e máximo de $\mathrm{R} \$$ 12.000,00.

Com relação aos hábitos de vida, predominaram os trabalhadores que não eram etilistas nem tabagistas, que referiram realização de atividades de lazer, não realização de atividade física regular, que tinham boa/muito boa qualidade de vida e bom/muito bom nível de saúde (Tabela 1).

A prevalência global de TMC foi $22,9 \%$. As estimativas das prevalências de TMC segundo características socioeconômicas e hábitos de vida mostrou que as prevalências foram mais elevadas entre as mulheres, entre os que não participavam de atividades de lazer nem realizavam atividade física, entre os que não tinham boa qualidade de vida e entre os trabalhadores com nível de saúde autorreferidos como regular, ruim ou muito ruim (Tabela 1). Essas variáveis apresentaram associações estatisticamente significantes com TMC nessa etapa da análise.

Com relação às características do trabalho predominaram os trabalhadores com vínculo permanente, com apenas um vínculo empregatício, que tinham até 40 horas de trabalho semanais, com até cinco anos no trabalho atual, que estavam satisfeitos com o trabalho, com a capacidade para desenvolvê-lo e que não se sentiam sufocados pela pressão do tempo. Com relação aos aspectos psicossociais do trabalho, 43,5\% referiram alta demanda psicológica e $47,9 \%$ baixo controle sobre o próprio trabalho (Tabela 2). Ressalta-se, na população estudada, que o predomínio do tipo de vínculo de trabalho estável deve-se à maior proporção de agentes comunitários de saúde (99,7\% com vínculo efetivo) e agentes de combate a endemias $(85,8 \%)$. Assinala-se, entretanto, que a maior parte dos demais profissionais possuía vínculo de trabalho temporário (100\% entre os profissionais do Nasf, 97,3\% entre os trabalhadores de enfermagem, 96,7\% entre os trabalhadores administrativos e 96,7\% entre os médicos).

Segundo o local de trabalho, a maior prevalência de TMC foi observada entre os trabalhadores do Nasf. Quando se analisou a categoria profissional, a menor prevalência de TMC foi entre os médicos $(7,3 \%)$ e a mais elevada foi observada entre os trabalhadores no Nasf $(31,6 \%)$, seguidos pelos agentes comunitários de saúde $(28,9 \%)$ (Tabela 2). 
Tabela 1 Prevalência de transtornos mentais comuns, razão de prevalência e intervalo de confiança segundo características sociodemográficas e hábitos de vida de trabalhadores da Atenção Básica - Feira de Santana, BA, 2012

\begin{tabular}{|c|c|c|c|c|c|}
\hline \multirow{2}{*}{ Variáveis (N) } & \multicolumn{2}{|c|}{ Frequência } & \multirow{2}{*}{$\frac{\text { Prev. }}{\%}$} & \multirow{2}{*}{$R P$} & \multirow{2}{*}{ IC $95 \%$} \\
\hline & $n$ & $\%$ & & & \\
\hline \multicolumn{6}{|l|}{ Sexo (762) } \\
\hline Feminino & 644 & 84,5 & 25,0 & 2,22 & $1,30-3,75^{*}$ \\
\hline Masculino & 118 & 15,5 & 11,3 & - & - \\
\hline \multicolumn{6}{|l|}{ Faixa etária (762) } \\
\hline$\leq 40$ anos & 442 & 58,0 & 24,1 & - & - \\
\hline 41 anos ou mais & 320 & 42,0 & 21,3 & 0,88 & $0,67-1,16$ \\
\hline \multicolumn{6}{|l|}{ Escolaridade (762) } \\
\hline Superior & 126 & 16,5 & 18,9 & - & - \\
\hline Outros & 636 & 83,5 & 23,7 & 1,26 & $0,85-1,86$ \\
\hline \multicolumn{6}{|l|}{ Situação conjugal (762) } \\
\hline Sem companheiro & 271 & 35,6 & 21,9 & 0,94 & $0,71-1,23$ \\
\hline Com companheiro & 491 & 64,4 & 23,4 & - & - \\
\hline \multicolumn{6}{|l|}{ Ter filhos (762) } \\
\hline Não & 227 & 29,8 & 22,1 & - & - \\
\hline $\operatorname{Sim}$ & 535 & 70,2 & 23,2 & 1,05 & $0,79-1,40$ \\
\hline \multicolumn{6}{|l|}{ Renda (536) } \\
\hline Até dois salários mínimos"*** & 641 & 84,1 & 23,7 & 1,29 & $0,86-1,93$ \\
\hline Acima de dois salários mínimos & 121 & 15,9 & 18,6 & - & - \\
\hline \multicolumn{6}{|l|}{ Consome bebida alcoólica (762) } \\
\hline Não & 586 & 76,9 & 24,0 & - & - \\
\hline Sim & 176 & 23,1 & 19,2 & 0,80 & $0,57-1,12$ \\
\hline \multicolumn{6}{|l|}{ Tabagismo (755) } \\
\hline Não fumante & 726 & 96,2 & 22,7 & - & - \\
\hline Fumante & 29 & 3,8 & 25,9 & 1,14 & $0,60-2,19$ \\
\hline \multicolumn{6}{|l|}{ Atividades de lazer (762) } \\
\hline Não & 80 & 10,5 & 36,4 & 1,70 & $1,22-2,37^{*}$ \\
\hline Sim & 682 & 89,5 & 21,4 & - & - \\
\hline \multicolumn{6}{|l|}{ Atividade física (689) } \\
\hline Não & 395 & 57,3 & 26,2 & 1,69 & $1,23-2,32^{*}$ \\
\hline Sim & 294 & 42,7 & 15,5 & - & - \\
\hline \multicolumn{6}{|l|}{ Qualidade de vida autorreferida (761) } \\
\hline Muito ruim/ruim/nem ruim, nem boa & 241 & 31,7 & 38,6 & 2,51 & $1,94-3,24 \%$ \\
\hline Boa/muito boa & 520 & 68,3 & 15,2 & - & - \\
\hline \multicolumn{6}{|l|}{ Nível de saúde autorreferido (761) } \\
\hline Bom/muito bom & 610 & 80,2 & 16,0 & - & - \\
\hline Regular/ruim/muito ruim & 151 & 19,8 & 51,0 & 3,19 & $2,51-4,07^{*}$ \\
\hline
\end{tabular}


Tabela 2 Prevalência de transtornos mentais comuns, razão de prevalência e intervalo de confiança segundo características do trabalho entre trabalhadores da Atenção Básica Feira de Santana, BA, 2012

\begin{tabular}{|c|c|c|c|c|c|}
\hline \multirow{2}{*}{ Variáveis (n) } & \multicolumn{2}{|c|}{ Frequência } & \multirow{2}{*}{$\frac{\text { Prev. }}{\%}$} & \multirow{2}{*}{$R P$} & \multirow{2}{*}{ IC 95\% } \\
\hline & $n$ & $\%$ & & & \\
\hline \multicolumn{6}{|l|}{ Tipo de vínculo (760) } \\
\hline Permanente & 475 & 62,5 & 24,1 & - & - \\
\hline Temporário & 285 & 37,5 & 12,4 & 0,52 & $0,33-0,79 *$ \\
\hline \multicolumn{6}{|l|}{ Outro vínculo (758) } \\
\hline Não & 629 & 83,0 & 20,5 & - & - \\
\hline Sim & 129 & 17,0 & 20,3 & 1,01 & $0,62-1,64$ \\
\hline \multicolumn{6}{|l|}{ Ocupação (762) } \\
\hline Médico & 43 & 5,6 & 7,3 & 1,00 & - \\
\hline Trabalhadores de enfermagem & 150 & 19,7 & 14,9 & 2,05 & $0,64-6,50$ \\
\hline Dentista/Agente de Saúde Bucal & 31 & 4,1 & 16,1 & 2,20 & $0,57-8,53$ \\
\hline Agentes Comunitários de Saúde & 340 & 44,6 & 28,9 & 3,95 & $1,31-11,88$ \\
\hline Trabalhadores do Nasf & 21 & 2,8 & 31,6 & 4,32 & $1,21-15,44$ \\
\hline Trabalhadores Vigilância Epidemiológica & 106 & 13,9 & 25,7 & 3,51 & $1,13-10,95$ \\
\hline Trabalhadores Administrativos & 71 & 9,3 & 16,9 & 2,31 & $0,69-7,71$ \\
\hline \multicolumn{6}{|l|}{ Jornada Semanal (762) } \\
\hline Até 40 horas & 651 & 85,4 & 10,0 & - & - \\
\hline Mais de 40 horas & 111 & 14,6 & 21,0 & 2,10 & $0,71-6,24$ \\
\hline \multicolumn{6}{|l|}{ Tempo de trabalho (751) } \\
\hline$\leq 5$ anos & 695 & 40,3 & 20,7 & - & - \\
\hline$>5$ anos & 56 & 59,7 & 19,9 & 0,96 & $0,69-1,35$ \\
\hline \multicolumn{6}{|l|}{ Satisfação com o trabalho (760) } \\
\hline Insatisfeito & 180 & 23,7 & 40,6 & 2,34 & $1,82-3,00^{*}$ \\
\hline Satisfeito & 580 & 76,3 & 17,4 & - & - \\
\hline \multicolumn{6}{|l|}{ Satisfação com a capacidade para o trabalho (760) } \\
\hline Insatisfeito & 150 & 19,7 & 45,3 & 2,62 & $2,04-3,35^{*}$ \\
\hline Satisfeito & 610 & 80,3 & 17,3 & - & - \\
\hline \multicolumn{6}{|l|}{ Pressão do tempo (759) } \\
\hline Não & 495 & 65,2 & 16,5 & - & - \\
\hline Sim & 264 & 34,8 & 35,0 & 2,12 & $1,64-2,75^{*}$ \\
\hline \multicolumn{6}{|l|}{ Demanda psicológica (752) } \\
\hline Baixa & 425 & 56,5 & 17,7 & - & - \\
\hline Alta & 327 & 43,5 & 28,8 & 1,61 & $1,23-2,10^{*}$ \\
\hline \multicolumn{6}{|l|}{ Controle sobre o trabalho (748) } \\
\hline Baixo & 358 & 47,9 & 27,3 & 1,44 & $1,10-1,89 *$ \\
\hline Alto & 390 & 52,1 & 18,9 & - & - \\
\hline
\end{tabular}

* p-valor $\leq 0,05$

Foi perdida informação para as seguintes variáveis: tipo de vínculo (2 trabalhadores), outro vínculo (4), tempo de trabalho (11), satisfação com o trabalho (2), satisfação com a capacidade para o trabalho (2), pressão do tempo (3), demanda psicológica (10) e controle sobre o próprio trabalho (14). 
Os profissionais do Nasf eram, predominantemente, do sexo feminino, com idade $\leq 40$ anos, sem companheiro, sem filhos, renda maior que dois salários mínimos, qualidade de vida boa/muito boa, com até cinco anos no trabalho atual, vínculo temporário, jornada de trabalho de $\leq 40$ horas semanais, com mais de um vínculo empregatício, consideravam o salário inadequado para o esforço que realizavam, tinham alta demanda e alto controle sobre o próprio trabalho e significativo percentual deles estava insatisfeito com o trabalho.
Com relação às características do trabalho, encontraram-se maiores prevalências de TMC entre os trabalhadores insatisfeitos com o trabalho, insatisfeitos com sua capacidade para o trabalho, sufocados pela pressão do tempo, com elevadas demandas psicológicas e baixo controle sobre o próprio trabalho (Tabela 2).

Na análise de TMC segundo os grupos do modelo demanda-controle encontrou-se prevalência mais elevada entre os que possuíam trabalho de alta exigência quando comparado com baixa exigência (Tabela 3).

Tabela 3 Prevalência de transtornos mentais comuns segundo os grupos do modelo demanda-controle, nos trabalhadores da Atenção Básica - Feira de Santana, BA, 2012

\begin{tabular}{lcccc}
\hline \multirow{2}{*}{ Categorias do Modelo Demanda-Controle } & \multicolumn{4}{c}{ Prevalência de TMC } \\
\cline { 2 - 5 } & $n$ & $\%$ & $R P$ & IC 95\% \\
\hline Trabalho passivo (baixo controle, baixa demanda) & 40 & 21,2 & 1,43 & $0,94-2,17$ \\
Trabalho ativo (alto controle, alta demanda) & 39 & 24,5 & 1,66 & $1,09-2,51$ \\
Alta exigência (baixo controle, alta demanda) & 53 & 33,3 & 2,25 & $1,53-3,31^{*}$ \\
Baixa exigência (alto controle, baixa demanda) & 33 & 14,8 & - & - \\
\hline
\end{tabular}

* $\mathrm{p}$-valor $\leq 0,05$

Após análise bivariada, foi realizada a análise multivariada (ARLM). As variáveis associadas aos TMC na análise bivariada foram avaliadas, simultaneamente, na ARLM. A Tabela 4 apresenta os dados do modelo final obtido.

No modelo final de análise, foram observadas associações estatisticamente significantes, segundo o critério definido $(\mathrm{p} \leq 0,05)$, de TMC com: sexo, qualidade de vida autorreferida, estado de saúde autorreferido, pressão do tempo, insatisfação com o trabalho e demanda psicológica. As situações com maior ocorrência de TMC foram sexo feminino, qualidade de vida autorreferida como muito ruim/ruim/ regular, estado de saúde autorreferido como regular/ruim/muito ruim, pressão do tempo, insatisfação com o trabalho e ter alta demanda psicológica.

Tabela 4 Estimativas do modelo selecionado na análise de regressão logística múltipla. Atenção Básica - Feira de Santana, BA, 2012

\begin{tabular}{|c|c|c|}
\hline Variáveis & $R P$ & IC 95\% \\
\hline \multicolumn{3}{|l|}{ Sexo } \\
\hline Feminino & 2,01 & $1,20-3,39^{*}$ \\
\hline Masculino & - & - \\
\hline \multicolumn{3}{|l|}{ Qualidade de vida autorreferida } \\
\hline Muito ruim/ ruim/ regular & 1,67 & $1,24-2,25$ 粠 \\
\hline Boa/muito boa & - & - \\
\hline \multicolumn{3}{|l|}{ Estado de saúde autorreferido } \\
\hline Regular/ ruim/ muito ruim & 2,28 & $1,69-3,07^{\text {米 }}$ \\
\hline Bom/muito bom & - & - \\
\hline \multicolumn{3}{|l|}{ Pressão do tempo } \\
\hline Sim & 1,47 & $1,11-1,95^{*}$ \\
\hline Não & - & - \\
\hline \multicolumn{3}{|l|}{ Satisfeito com o trabalho } \\
\hline Insatisfeito & 1,26 & $1,04-1,52^{*}$ \\
\hline Satisfeito & - & - \\
\hline \multicolumn{3}{|l|}{ Demanda psicológica } \\
\hline Alta & 1,35 & $1,03-1,78^{* * * *}$ \\
\hline Baixa & & \\
\hline
\end{tabular}




\section{Discussão}

A prevalência global de TMC foi de $22,9 \%$, sendo mais elevada do que a encontrada em outros grupos ocupacionais como motoristas e cobradores de ônibus da cidade de São Paulo $(20,3 \%)^{22}$ e industriários, $11,6 \%{ }^{23}$, o que evidencia que as questões relativas à saúde mental são muito relevantes nesse grupo de trabalhadores. Portanto, esses achados reforçam os resultados de elevadas prevalências de TMC entre trabalhadores da saúde quando comparados a outras categorias de trabalhadores. Além disso, são achados próximos a outros estudos com categorias específicas de profissionais de saúde, como médicos e enfermeiras. Entre os médicos de Salvador (BA), observou-se prevalência global de TMC de $26 \%$, aumentando para $34,6 \%$ para o sexo feminino ${ }^{24}$. Entre trabalhadoras de enfermagem de um Hospital Geral de Salvador, a prevalência de TMC foi de $33,3 \%{ }^{2}$.

O nível de adoecimento psíquico encontrado neste estudo foi inferior ao estudo realizado com trabalhadores da rede básica de saúde de Botucatu $(42,6 \%)^{7}$ e superior ao encontrado no estudo de trabalhadores da atenção básica nas regiões sul e nordeste do Brasil $(16,0 \%)^{25}$. Características específicas do contexto no qual os trabalhadores estão inseridos podem justificar as diferenças observadas. O uso de diferentes pontos de corte para definição de transtorno mental também é outro fator que pode interferir nos resultados obtidos, comprometendo a comparação desses achados ${ }^{17}$.

A descrição de dados de frequências de TMC em diferentes grupos ocupacionais e categorias profissionais da saúde ajuda a dimensionar a relevância do sofrimento psíquico entre os trabalhadores estudados, com base em estimativas de transtornos identificados em diferentes contextos. Portanto, é um dado que deve ser visto com cautela uma vez que não estão sendo discutidos ou comparados aspectos específicos dos diferentes tipos de trabalho citados. Cabe ainda assinalar que a ocorrência de TMC em quase um quarto da população investigada é um indicador que fala por si mesmo, explicitando a necessidade de atenção, intervenção e acompanhamento da saúde mental da população atingida.

Considerando-se como variável de exposição principal o local de trabalho, o grupo de maior vulnerabilidade foi o de trabalhadores do Nasf, resultado semelhante ao obtido no estudo de Botucatu ${ }^{7}$, chamando atenção para a necessidade de avaliação mais atenta desse grupo profissional e de suas demandas de trabalho.

O trabalho do Nasf é desenvolvido por diversos profissionais de nível superior, que devem apoiar os profissionais das Equipes de Saúde da Família
(ESF) no atendimento às demandas do território, por meio do encaminhamento com base na referência e contrarreferência, com vistas a desenvolver acompanhamento da população assistida. Cada núcleo é responsável por um número de unidades de ESF, oferecendo apoio matricial e construindo articulações com os serviços de referência existentes. O Nasf deve contar com pelo menos um profissional da área de saúde mental devido à magnitude epidemiológica dos transtornos mentais existentes na população. Trata-se de um tipo de trabalho predominantemente educativo, baseado em atividades em grupo e de orientação, o que pode estar interferindo na maior prevalência de TMC neste grupo, posto que, além da sobrecarga de trabalho, é difícil visualizar os resultados da ação de seu trabalho em curto prazo, além de possuírem vinculo de trabalho precário, realizado por cooperativas, sem as garantias trabalhistas básicas como férias e décimo terceiro salário ${ }^{7}$. Nos Nasf estudados, como já mencionado, todos os profissionais tinham vínculo de trabalho temporário, realizado por intermédio de cooperativa.

Além disso, esses trabalhadores vivenciam, na prática diária, níveis excessivos de responsabilidades somados ao tempo insuficiente para planejar e preparar a assistência, além de interrupções constantes. Estudos com pessoal de enfermagem evidenciam que essas características são geradoras de transtornos mentais, especialmente quando se tem contato direto com a dor e o sofrimento dos doentes ${ }^{26}$.

Deve-se atentar também para a elevada prevalência de TMC entre os Agentes Comunitários de Saúde (ACS), segundo grupo de maior prevalência na população estudada. Maior vulnerabilidade dos ACS foi também encontrada no estudo entre trabalhadores da atenção primária do sul e nordeste do Brasil ${ }^{25}$, registrando-se nesse grupo maior adoecimento psíquico quando comparado aos demais profissionais da atenção básica estudados.

As mulheres apresentaram maior prevalência de TMC do que os homens - resultado consistentemente encontrado em outros estudos de profissionais de saúde ${ }^{2,24,27}$. Possíveis explicações para esse resultado estão relacionadas ao duplo papel que as mulheres vêm desempenhando na sociedade. As mudanças ocorridas nas últimas décadas têm mostrado que, além de exercerem as funções oriundas de sua inserção no mercado de trabalho, as mulheres são também as principais responsáveis pelas tarefas domésticas e educação dos filhos, o que aumenta suas demandas e a carga total de trabalho ${ }^{28,29}$.

A segregação da força de trabalho segundo o sexo e o persistente desequilíbrio das relações de gênero no trabalho doméstico condicionam as trabalhadoras à dupla exposição: fatores de risco ocupacionais e os da vida doméstica, sendo 
esta de elevada sobrecarga, com impacto no tempo para lazer e repouso. Demandas contínuas com diminuição do tempo para descanso e relaxamento implicam dificuldades de recuperação do organismo e aumentam as chances de ocorrência dos sintomas musculoesqueléticos, desencadeados pelas atividades laborais e transtornos mentais ${ }^{2,30}$.

A qualidade de vida foi outro aspecto associado, neste estudo, aos TMC. Em estudo realizado em Pelotas (RS) observou-se associação entre TMC e qualidade de vida ${ }^{31}$, confirmando que o trabalho em conjunto com a alimentação, a moradia e a educação, constituem necessidades humanas básicas a serem atendidas com o intuito de edificar uma vida com qualidade $^{32}$.

Ter nível de saúde autorreferido como regular/ ruim/muito ruim associou-se à maior prevalência de TMC, indicando que o trabalhador da atenção básica necessita de atenção quanto a sua saúde física e mental. Em pesquisa realizada com ACS de um município do Paraná, o nível de saúde foi considerado intermediário para 15,4\% dos participantes; 3,6\%, como desfavorável (fraco ou muito ruim) e 43,8\% relataram ter algum problema de saúde ${ }^{13}$. Portanto, uma vez que se têm observado elevados percentuais de baixos níveis de saúde entre os trabalhadores da atenção primária, reforça-se a necessidade de implementação de mudanças nas condições e organização do trabalho, bem como a criação de programas de acompanhamento e monitoramento da situação de saúde dos trabalhadores. Os exames médicos periódicos, incluindo a análise da saúde mental, constituem medidas relevantes nessa direção.

A insatisfação com o trabalho também vem sendo evidenciada entre profissionais de saúde ${ }^{33}$. A necessidade de oferecer serviços adequados para garantir a saúde como direito do cidadão sem, muitas vezes, possuir os mínimos meios para realizá-los adequadamente, somado ao fator tempo e à dificuldade estrutural da atenção básica do país (como a insuficiência de outros níveis de atenção e de ações intersetoriais, além das distorções entre necessidade e consumo de serviços de saúde e da falta de discussão sobre plano de cargos e salários para os trabalhadores), tem gerado desgaste profissional e insatisfações ${ }^{5,7,34,35}$.

Associação entre insatisfação com o trabalho e prevalência de TMC foi encontrada na Atenção Básica de Botucatu ${ }^{7}$. Estudo com médicos da atenção primária de Belo Horizonte (MG) também evidenciou associação positiva entre a insatisfação com o trabalho e $\mathrm{TMC}^{36}$. A satisfação no trabalho exerce influência no estado emocional do indivíduo, manifestando-se por sentimentos de alegria e contentamento, relacionando-se a como o indivíduo percebe, valoriza e julga os aspectos do trabalho ${ }^{37}$.
A satisfação no trabalho pode relacionar-se às taxas de rotatividade ou de permanência no trabalho. Trabalhadores que não estão realizados profissionalmente, que recebem pouco reconhecimento no cargo, vivenciam constantes conflitos com a chefia ou colegas ou não atingiram o patamar desejado em suas carreiras, tendem a apresentar maior rotatividade no emprego e maior nível de adoecimento físico e mental ${ }^{33,38}$.

Aspectos como a possibilidade de controlar as atividades que realiza, em termos da planificação e determinação de procedimentos a utilizar, bem como em termos de salário, benefícios e tempo de trabalho estão diretamente ligados ao cotidiano laboral e influenciam a satisfação com o trabalho e o bem-estar psicológico ${ }^{39}$.

Outro elemento associado ao adoecimento psíquico foi a pressão do tempo. Essa condição, além de ser um indicador de intensificação do trabalho (as tarefas devem ser feitas em um ritmo mais acelerado do que o necessário para seu desempenho satisfatório) implica também ausência de autonomia do trabalhador sobre o próprio trabalho. A pressão do tempo impõe um ritmo próprio de realização das atividades laborais, assim, não permite que o trabalhador defina como realizar seu trabalho ou adequar sua execução aos parâmetros e expectativas que ele possui. Esses elementos aumentam a tensão sobre o trabalhador, gerando sofrimento e adoecimento.

A literatura registra evidências consistentes de associação entre elevadas demandas psicológicas no trabalho e transtornos mentais comuns entre trabalhadores de saúde $2,24,27,36$, como foi observado neste estudo. Demandas laborais excessivas sem a correspondente possibilidade de recuperação ou relaxamento, que favoreceriam o retorno do corpo à situação de acalmia, mantêm a produção elevada das catecolaminas (adrenalina, noradrenalina e cortisol), produzindo intenso desgaste físico e mental ${ }^{18}$. Desse modo, configuram situações desfavoráveis aos indivíduos, gerando adoecimento. No setor saúde, essas demandas possuem forte conteúdo emocional, uma vez que se está lidando com sofrimento, doença e morte das pessoas. Assim, demandas excessivas podem promover sentimentos de inadequação e incapacidade entre os trabalhadores, elevando seu próprio sofrimento e vulnerabilidade.

Desse modo, as elevadas cargas de trabalho a que estão submetidos os trabalhadores da saúde podem gerar potenciais agravos que, ao longo dos anos, alteram a saúde dos trabalhadores, dificultam seu relacionamento na equipe de trabalho, reduzem a produtividade, favorecem o adoecimento físico e mental, necessitando, portanto, ser identificadas e prevenidas e/ou enfrentadas precocemente, 
especialmente pelo desgaste físico que acarreta sofrimento psíquico ${ }^{5}$.

Menor prevalência de TMC foi observada entre os trabalhadores com vínculo de trabalho temporário. Assim, cabe reflexão sobre as características do emprego no setor saúde nos contextos atuais. Embora a literatura aponte que vínculos estáveis de trabalho constituam situações mais protegidas para os trabalhadores, tem-se observado, no setor saúde, mudanças na conformação das características dos vínculos de emprego e trabalho que alteram, e tornam complexas, essa situação esperada ${ }^{35,40}$.

Dois elementos devem ser considerados na análise desses resultados. O primeiro deles é o estabelecimento de contratos temporários como a forma principal de contratação dos trabalhadores com maior escolaridade/qualificação e salários mais elevados (caso dos médicos, dentistas e enfermeiros). Os estudos apontam que a maior parte dos trabalhadores da atenção primária nessas profissões mantém vínculos de trabalho temporário ${ }^{41}$; resultado também encontrado neste estudo. O segundo elemento, relacionado ao primeiro, refere-se à composição dos trabalhadores com vínculo de trabalho estável, com o predomínio dos agentes comunitários de saúde (ACS). Para esse grupo, há a exigência legal de que essa ocupação seja preenchida por meio de concurso público. No entanto, as condições de trabalho desses trabalhadores têm sido apontadas como precárias, estando os ACS submetidos a vários tipos de exposição ocupacional, a baixos salários e baixo reconhecimento social $^{5,7,34}$ - elementos associados em vários estudos ao adoecimento psíquico ${ }^{2,42}$. Ou seja, os ACS possuem estabilidade no emprego, mas trabalham em condições precárias, recebendo os mais baixos salários. Observa-se, assim, dissociação entre a garantia de estabilidade no emprego e acesso aos direitos trabalhistas e a qualificação/melhor remuneração do trabalho no setor saúde. Essa lógica de funcionamento afeta também a perspectiva de coletivo entre os trabalhadores da saúde, uma vez que fragmenta e separa os interesses dos grupos em seu interior.

Portanto, essas questões mostram a complexidade das relações de emprego e trabalho existentes no setor saúde na atualidade e os múltiplos elementos envolvidos em sua análise. Achados como esses mostram que estão configuradas novas situações, que mesclam situações anteriores bastante distintas, tanto de conformação de vínculos quanto de características desses vínculos e da composição do perfil profissional dos tipos existentes. Portanto, esses aspectos devem ser explorados em maior profundidade em estudos futuros de modo a possibilitar maior compreensão sobre as relações observadas.
Este estudo gerou um conjunto de resultados importantes que podem contribuir para a reflexão e o redesenho dos modelos de gestão do trabalho na Atenção Básica. No entanto, na análise de seus resultados, também devem ser consideradas suas limitações. Uma limitação relevante refere-se ao delineamento de pesquisa deste estudo. Como foi adotada metodologia de desenho transversal, que investiga simultaneamente causa e efeito (exposição e desfecho), não é possível avaliar relações diretas de causalidade entre as variáveis estudadas; ou seja, a investigação feita não permite afirmar se os aspectos associados aos TMC, de fato, produziram TMC, ou se os trabalhadores com TMC tornaram-se mais vulneráveis aos aspectos investigados. Observou-se apenas que esses eventos ocorreram simultaneamente. Portanto, o efeito de causalidade reversa não pode ser descartado neste caso. Outra possível limitação decorre do chamado efeito do trabalhador sadio. Como se trata de uma investigação no local de trabalho, incluindo os trabalhadores em efetiva atividade no momento da coleta dos dados, é possível que os trabalhadores doentes, que estavam afastados de suas atividades, não tenham sido estudados, o que pode subestimar a prevalência de transtornos mentais nesta população.

Apesar dessas limitações, o estudo avaliou amostra representativa da população-alvo, selecionada de modo aleatório, garantindo-se a possibilidade de que qualquer indivíduo, em efetiva atividade laboral, pudesse ser selecionado para o estudo. Assim, o estudo tem uma boa validade externa. Adicionalmente também avaliou uma gama ampla de aspectos da vida e do trabalho (características sociodemográficas e do trabalho, hábitos de vida, qualidade de vida, situação de saúde e aspectos psicossociais do trabalho). Desse modo, os resultados obtidos podem fornecer informações úteis e válidas para orientar medidas e ações de proteção à saúde dos trabalhadores, abarcando diversas dimensões de suas vidas.

A análise dos transtornos mentais comuns entre os trabalhadores da Atenção Básica mostrou maior prevalência entre os trabalhadores do Nasf, tanto ao analisar esse dado por local de trabalho quanto por categoria profissional. As mulheres, a qualidade de vida autorreferida muito ruim/ruim/regular, o estado de saúde autorreferido como regular/ruim/muito ruim, a pressão do tempo, insatisfação com o trabalho e alta demanda psicológica foram fatores associados à maior prevalência de TMC.

Esses dados evidenciam a necessidade de atenção especial a esse grupo de trabalhadores, bem como de outros estudos com essa categoria. Desse modo, esses aspectos constituem elementos de atenção especial para a elaboração e implementação de medidas de intervenção nesse grupo. 


\section{Contribuições de autoria}

Carvalho DB e Araújo TM: contribuição substancial no projeto e delineamento, no levantamento de dados e em sua análise e interpretação; elaboração do manuscrito e contribuição importante em sua revisão crítica e aprovação final da versão publicada. Bernardes KO: contribuição substancial no projeto e delineamento, no levantamento de dados e em sua análise.

\section{Referências}

1. Organização Mundial de Saúde. Mistério da saúde. Relatório Mundial de Saúde - Saúde mental: nova concepção, nova esperança. Genebra: OMS; 2002.

2. Araújo TM, Aquino E, Menezes G, Santos CO, Aguiar L. Aspectos psicossociais do trabalho e distúrbios psíquicos entre trabalhadoras de enfermagem. Rev Saúde Pública. 2003;4(37):424-33.

3. Silva-Junior JS, Fischer FM. Long-term sickness absence due to mental disorders is associated with individual features and psychosocial work conditions. PLoS One, 2014;9(12):e115885.

4. Sala A, Parreira SMCP. O trabalho no setor hospitalar: adoecimentos e afastamentos por motivos de saúde. In: Assunção AA, Brito J, organizadores. Trabalhar na saúde: experiências cotidianas e desafios para a gestão do trabalho e do emprego. Rio de Janeiro: Fiocruz; 2011. p. 151-70.

5. Trindade LL, Gonzales RMB, Beck CLC, Lautert L. Cargas de trabalho entre os agentes comunitários de saúde. Rev Gaúcha Enferm. 2007;4(28):473-79.

6. Ramminger T. A saúde mental do trabalhador em saúde mental: um estudo com trabalhadores de um hospital psiquiátrico. Bol saúde. 2002;16(1):111-24.

7. Braga LC, Carvalho LR, Binder MCP. Condições de trabalho e transtornos mentais comuns em trabalhadores da rede básica de saúde de Botucatu (SP). Ciênc Saúde Coletiva. 2010;15(Supl 1):1585-96.

8. Brasil. Ministério da Saúde. Secretaria de Atenção à Saúde. Departamento de Atenção Básica. Política Nacional de Atenção Básica. 1a ed. Série Pactos pela Saúde. Brasília, DF; 2006. vol. 4.

9. Cunha JB, Blank VLG, Boing AF. Tendência temporal de afastamento do trabalho em serviços públicos (1995-2005). Rev Bras Epidemiol. 2009;12(2):226-36.

10. Nascimento DDG, Oliveira CMA. Reflexões sobre as competências profissionais para o processo de trabalho nos Núcleos de Apoio à Saúde da Família. Mundo saúde. 2010;34(1):92-6.

11. Leite DF, Nascimento DDG, Oliveira, MAC. Qualidade de vida no trabalho de profissionais do Nasf no município de São Paulo. Physis. 2014;24(2):507-25.

12. Silva ATC, Menezes PR. Esgotamento profissional e transtornos mentais comuns em agentes comunitários de saúde. Rev Saúde Pública. 2008;42(5):921-9.
13. Kluthcovsky ACGC, Takayanagui AMM, Santos CB, Kluthcovsky FA. Avaliação da qualidade de vida geral de agentes comunitários de saúde: a contribuição relativa das variáveis sociodemográficas e dos domínios da qualidade de vida. Rev psiquiatria Rio Gd Sul. 2007;29(2):176-83.

14. Rocha PM, Uchoa AC, Rocha NSPD, Souza ECF, Rocha ML, Pinheiro TXA. Avaliação do Programa Saúde da Família em municípios do Nordeste brasileiro: velhos e novos desafios. Cad Saúde Pública. 2008;24(Supl 1):S69-S78.

15. Instituto Brasileiro de Geografia e Estatística. Estimativas da população dos municípios brasileiros com data de referência em $1^{\circ}$ de julho de 2014. 2014 [citado em 2014 ago 28]. Disponível em: http://bit.ly/1U8mvLT.

16. Feira de Santana. Secretaria Municipal de Saúde. Estabelecimentos de Saúde, 2012 [citado em 2012 ago 26]. Disponível em: http://bit.ly/2fyI24V.

17. Santos KOB, Araújo TM, Oliveira NF. Estrutura fatorial e consistência interna do Self-Reporting Questionnaire (SRQ-20) em população urbana. Cad Saúde Pública. 2009;25(1):214-22.

18. Karasek R, Theörell T. Healthy work-stress, productivity, and the reconstruction of working life. New York: Basic Books; 1990.

19. Santos KOB, Araújo TM, Pinho PS, Silva ACC. Avaliação de um instrumento de mensuração de morbidade psíquica: estudo de validação do Self-Reporting Questionnaire (SRQ-20). RBSP. 2010;34(3):544-56.

20. Hosmer DW, Lemeshow S. Applied logistic regression. 2a ed. New York: John Wiley e Sons; 2000.

21. Oliveira NF, Santana VS, Lopes AA. Razões de proporções e uso do método Delta para intervalos de confiança em regressão logística. Rev Saúde Pública. 1997;31(1):90-99.

22. Souza MFM, Silva GR. Risco de distúrbios psiquiátricos menores em área metropolitana na região Sudeste do Brasil. Rev Saúde Pública. 1998;32(1):50-8.

23. Fonseca ISS, Araújo TM. Prevalência de transtornos mentais comuns em industriários da Bahia. Rev Bras Saúde Ocup. 2014;39(129):35-49.

24. Nascimento Sobrinho CL, Carvalho FM, Bonfim TAS, Cirino CAS, Ferreira IS. Condições de trabalho e saúde mental dos médicos de Salvador, 
Bahia, Brasil. Cad Saúde Pública. 2006;22(1):13140.

25. Dilelio AS, Faachini LA, Tomasi E, Silva SM, Thumé E, Piccini RX, et al. Prevalência de transtornos psiquiátricos menores em trabalhadores da atenção primária à saúde das regiões Sul e Nordeste do Brasil. Cad Saúde Pública. 2012;28(3):503-14.

26. Demerouti E, Bakker AB, Nachreiner F, Schaufeli WB. A model of burnout and life satisfaction amongst nurses. J Adv Nurs. 2000;32(2):454-64.

27. Kirchhof ALC, Magnago TSBS, Camponogara S, Griep RH, Tavares JP, Prestes FC, Paes LG. Condições de trabalho e características sociodemográficas relacionadas à presença de distúrbios psíquicos menores em trabalhadores de enfermagem. Texto contexto - enfermagem. 2009;18(2):215-23.

28. Araújo TM, Rotenberg L. Relações de gênero no trabalho em saúde: a divisão sexual do trabalho e a saúde dos trabalhadores. In: Assunção AA, Brito J, organizadores. Trabalhar na Saúde: experiências cotidianas e desafios para a gestão do trabalho e do emprego. Rio de Janeiro: Fiocruz; 2011. p. 131-150.

29. Rocha SV, Araújo TM, Almeida MMG, Virtuoso Júnior JS. Prática de atividade física no lazer e transtornos mentais comuns entre residentes de um município do Nordeste do Brasil. Rev Bras de Epidemiol. 2012;15(4):871-83.

30. Strazdins L, Bammer G. Women, work and musculoskeletal health. Soc Sci Med. 2004;58(6):997-1005.

31. Jansen K, Mondin TC, Ores LC, Souza LDMS, Konradt CE, Pinheiro RT, et al. Transtornos mentais comuns e qualidade de vida em jovens: uma amostra populacional de Pelotas, Rio Grande do Sul, Brasil. Cad Saúde Pública. 2011;27(3):440-48.

32. Nahas MV. Atividade física, saúde e qualidade de vida: conceitos e sugestões para um estilo de vida ativo. Londrina: Midiograf; 2001.
33. Ribeiro RN, Assunção AA, Araújo TM. Factors Associated with Job Satisfaction among PublicSector Physicians in Belo Horizonte, Brazil. Int J Health Serv. 2014;44(4):787-804.

34. Maia LDG, Silva ND, Mendes PHC. Síndrome de Burnout em agentes comunitários de saúde: aspectos de sua formação e prática. Rev Bras Saúde Ocup. 2011;36(123):93-102.

35. Medeiros CRG, Junqueira AGW, Schwingel G, Carreno I, Jungles LAP, Saldanha OMFL. A rotatividade de enfermeiros e médicos: um impasse na implementação da Estratégia de Saúde da Família. Ciênc Saúde Coletiva. 2010;15(Supl 1):1521-31.

36. Assunção AA, Machado CJ, Prais HA, Araújo TM. et al. Working conditions and common mental disorders in physicians in Brazil. Occup Med. 2013;63(3):234-7.

37. Locke EA. What is job satisfaction? Organ Behav Hum Perform. 1969;4(4):309-36.

38. Davis K, Newstrom JW. Comportamento humano no trabalho: uma abordagem psicológica. São Paulo: Pioneira Thomson Learning; 2001.

39. Silva JLL. Estresse e transtornos mentais comuns em trabalhadores de enfermagem [dissertação]. Rio de Janeiro: Universidade Estadual do Rio de Janeiro; 2007.

40. Assunção AA. Condições de trabalho e saúde dos trabalhadores da saúde. In: Minayo C, Machado JMH, Pena PGL, organizadores. Saúde do trabalhador na sociedade brasileira contemporânea. Rio de Janeiro: Fiocruz; 2010. p. 453-478.

41. Barbosa G, Correia AKS, Oliveira LMM, Santos VC Ferreira SMSF, Martins Júnior DF, et al. Trabalho e saúde mental dos profissionais da Estratégia Saúde da Família em um município do Estado da Bahia, Brasil. Rev Bras Saúde Ocup. 2012;37(126):306-15.

42. Glina DMR, Rocha LE. Prevenção para a saúde mental no trabalho. Saúde mental no trabalho: desafios e soluções. São Paulo: VK; 2000. p. 53-82. 